\title{
вMJ Global Health Community-based HIV self-testing: a cluster-randomised trial of supply-side financial incentives and time-trend analysis of linkage to antiretroviral therapy in Zimbabwe
}

\author{
Euphemia Lindelwe Sibanda (D) ,, Melissa Neuman (D) , ${ }^{3}$ Mary Tumushime, ${ }^{1}$ \\ Collin Mangenah (D) , Karin Hatzold, ${ }^{4}$ Constancia Watadzaushe, ${ }^{1}$ \\ Miriam N Mutseta, ${ }^{5}$ Jeffrey Dirawo, ${ }^{1}$ Sue Napierala, ${ }^{6}$ Getrude Ncube, ${ }^{7}$ \\ Fern Terris-Prestholt, ${ }^{8}$ Miriam Taegtmeyer, ${ }^{2}$ Cheryl Johnson, ${ }^{9}$ \\ Katherine L Fielding, ${ }^{10}$ Helen A Weiss, ${ }^{10}$ Elizabeth Corbett, ${ }^{11,12}$ \\ Frances M Cowan (D) 1,2
}

To cite: Sibanda EL, Neuman M, Tumushime M, et al. Community-based HIV self-testing: a clusterrandomised trial of supplyside financial incentives and time-trend analysis of linkage to antiretroviral therapy in Zimbabwe. BMJ Global Health 2021;6:e003866. doi:10.1136/ bmjgh-2020-003866

Handling editor Edwine Barasa

- Additional material is published online only. To view, please visit the journal online (http://dx.doi.org/10.1136/ bmjgh-2020-003866).

Received 3 September 2020 Revised 10 February 2021 Accepted 18 February 2021

Check for updates

(c) World Health Organization 2021. Licensee BMJ.

For numbered affiliations see end of article.

Correspondence to Euphemia Lindelwe Sibanda; euphemia@ceshhar.co.zw

\section{ABSTRACT}

Background HIV self-testing (HIVST) requires linkage to post-test services to maximise its benefits. We evaluated effect of supply-side incentivisation on linkage following community-based HIVST and evaluated time-trends in facility-based antiretroviral therapy (ART) initiations.

Methods From August 2016 to August 2017 community-based distributors (CBDs) in 38 rural Zimbabwean communities distributed HIVST doorto-door in 19-25 day campaigns. Communities were allocated (1:1) using constrained randomisation to either one-off US $\$ 50$ remuneration per CBD (nonincentive arm), or US $\$ 50$ plus US $\$ 0.20$ incentive per client visiting mobile-outreach services (conditionalincentive arm). The primary outcome, assessed by population survey 6 weeks later, was self-reported uptake of any clinic service, analysed with randomeffects logistic regression. Separately, non-randomised difference-in-differences in monthly ART initiations were analysed for three time periods (6 months baseline; HIVST campaign; 3 months after) at public clinics with (40 clinics) and without (124 clinics) HIVST distribution in catchment area.

Findings $A$ total of 445 conditional-incentive CBDs distributed 39205 HIVST kits (mean/CBD: 88; $95 \%$ Cl: 85 to 92) and 447 non-incentive CBDs distributed 41173 kits (mean/CBD: 93; 95\% Cl: 89 to 96$)$. Survey participation was $7146 / 8566(83.4 \%)$, with $3593(50.3 \%)$ reporting self-testing including $1305(18.3 \%)$ previously untested individuals. Use of clinic services post-HIVST was similar in conditionalincentive $(1062 / 3698,28.7 \%)$ and non-incentive $(1075 / 3448,31.2 \%)$ arms (adjusted risk ratio (aRR) $0.94,95 \%$ Cl: 0.86 to 1.03$)$. Confirmatory testing by newly diagnosed/untreated HIVST+clients was, however, higher (conditional-incentive: 25/33, 75.8\% vs non-incentive: $20 / 40,50.0 \%$ : aRR: $1.59,95 \% \mathrm{Cl}$ :

\begin{abstract}
WHAT IS ALREADY KNOWN?
$\Rightarrow$ HIV self-testing increases uptake of HIV testing using a range of delivery models.

$\Rightarrow$ Linking to post-test services will increase benefits and cost effectiveness of HIV self-testing.

$\Rightarrow$ Demand-side incentives increase linkage to posttest services but the role of supply-side incentives is less clear.

$\Rightarrow$ We investigated whether supply-side incentives increase linkage to post-test services during community-based distribution of HIV self-test kits.

\section{WHAT ARE THE NEW FINDINGS?}

$\Rightarrow$ Community-based, door-to-door distribution of HIV self-test kits improved testing coverage.

$\Rightarrow$ Supply-side incentives for test kit distributors did not increase post-test service uptake.

$\Rightarrow$ In a post-hoc analysis supply-side incentives for HIV self-test kit distributors increased uptake of confirmatory testing among newly diagnosed HIV positive self-testers.

$\Rightarrow$ Community-based, door-to-door distribution of HIV self-tests was associated with a $27 \%$ increase in antiretroviral therapy initiations at nearby health facilities.

\section{WHAT DO THE NEW FINDINGS IMPLY?}

$\Rightarrow$ Community-based, door-to-door delivery of HIV selftest kits is feasible and scalable.

$\Rightarrow$ Future research should aim to optimise the nature and size of incentives to increase uptake and linkage while weighing the benefits and costs.
\end{abstract}

1.05 to 2.39). In total, 12808 ART initiations occurred, with no baseline or postcampaign differences between initiation rates in HIVST versus non-HIVST clinics, but initiation rates increased from 7.31 to 9.59 initiations 
per month in HIVST clinics during distribution, aRR: $1.27,95 \% \mathrm{Cl}$ 1.17 to 1.39 .

Conclusions Community-based HIVST campaigns achieved high testing uptake, temporally associated with increased demand for ART. Small supply-side incentives did not affect general clinic usage but may have increased confirmatory testing for newly diagnosed HIVST positive participants.

Trial registration number PACTR201607001701788.

\section{INTRODUCTION}

HIV testing services (HTS) are a key entry point for HIV prevention and treatment services. Despite major investment in HTS, an estimated $21 \%$ of people living with HIV (PLHIV) globally were unaware of their status in 2018. ' HIV self-testing (HIVST), whereby an individual uses a rapid diagnostic test to collect their own sample (oral or blood), performs the test and interprets the result, is recommended by WHO. ${ }^{2}$ It is highly acceptable, ${ }^{3-7}$ safe and can be highly accurate. ${ }^{8-10}$ Adding HIVST to clinic-based HTS can increase coverage and frequency of HIV testing, including among those less well-served by more traditional HTS approaches, such as rural populations, men, young people and key populations (sex workers, men having sex with men, transgender people, prisoners and people who inject drugs). ${ }^{11-15}$ Projections suggest that $4.6 \%$ of all 360 million HIV tests used each year globally will be self-tests by 2020, with higher percentages of $10 \%-35 \%$ in Kenya, Malawi, Zambia, Zimbabwe and South Africa. ${ }^{16}$

A reactive (positive) HIVST result needs confirmatory testing to provide an HIV-positive diagnosis followed by prompt linkage into HIV care. ${ }^{2}$ A negative HIVST result (which does not need confirmatory testing) provides an opportunity for HIV prevention, such as voluntary medical male circumcision (VMMC), pre-exposure prophylaxis and use of condoms. Ensuring linkage following HIVST is essential for maximising health outcomes and costeffectiveness. ${ }^{17}$ Evidence to date has been limited, but trials suggest that supportive linkage strategies are needed, including home-based antiretroviral therapy (ART) initiation ${ }^{18}$ and financial incentives provided to HIVST clients, ${ }^{19}$ among other strategies. ${ }^{20}$ However, the role of financial incentives for providers ('supplyside' incentives) has not previously been investigated for effectiveness on the later stages of the HIV care cascade (linkage and retention) (The HIV care cascade is the series of stages an HIV positive person goes through from HIV testing through to treatment initiation and viral suppression). ${ }^{21}$

The formative work that we conducted in nontrial sites showed that community-based HIVST is an acceptable and feasible model for providing HIVST. ${ }^{4}$ Here we report results from a cluster-randomised trial investigating conditional supply-side incentives given to lay community-based distributors (CBDs) to promote linkage to services following HIVST.

\section{METHODS}

\section{Population and setting}

We conducted the study in Zimbabwe, a country with an HIV prevalence of $14.1 \% .^{12}$ At the time of the study, Zimbabwe had already adopted HIVST into policy although self-tests were not yet widely available. Eight districts took part: Buhera, Bulilima, Chivi, Gutu, Gweru, Masvingo, Mazowe and Mberengwa, where the study unit was a ward (subdistrict administrative unit). We used administrative maps of the districts (online supplemental appendix 1) to select 44 out of a possible 254 wards with geographic separation. The number of districts was informed by the sample size: we continued to include new districts until we reached 44 eligible wards.

Starting with a randomly selected ward at the boundary of the district, we systematically worked through each map to select additional wards while ensuring that each was at least $15 \mathrm{~km}$ away from its nearest neighbour/s. The districts were chosen because of the presence of Population Services International (PSI) outreach clinics that deliver services, including standard HIV testing services, screening for TB, diabetes and hypertension, family planning and VMMC (Implementation of HIVST distribution was carried out by Unitaid/PSI as part of the Self Testing AfRica Initiative ${ }^{22}$ ). Within each district, there were public sector primary health clinics providing a range of services including provider-delivered HIV testing and treatment services. PSI worked closely with these facilities to prepare them to receive individuals who had selftested and were linking for different services according to self-test results.

\section{HIVST intervention in study communities (wards)}

PSI Zimbabwe identified, recruited and trained CBDs from each ward to distribute HIVST kits. Selection of CBDs was informed by community leaders, who recommended individuals whom they felt were suitable distributors based on previous experience of working with HIV programmes, community work, literacy levels and good standing in the community, hence CBDs were a heterogeneous group with different backgrounds and levels of experience. PSI provided CBDs with a 2-day training in HIV testing, supporting others to use HIVST kits, providing information to promote and support linkage to appropriate post-test services, use of data capture tools and the study protocol. Each village (smallest subunit of a ward that varies in size but typically has about 100 households) had one or two CBDs depending on size. Over four to 4-6 weeks, CBDs conducted door-to-door visits offering oral fluid self-test kits to all household members $\geq 16$ years old (the Ministry of Health-prescribed minimum age for consenting to HIV testing). Kits were only given to individuals providing verbal consent for self-testing. CBDs recorded distribution data onto computer tablets. They 
advised clients to seek further HIV services as needed from PSI outreach clinics and/or local primary clinics. Kit recipients were given a toll-free helpline number that they could use as needed.

PSI staff provided outreach clinic services described above (Population and Setting) soon after starting and completing HIVST distribution, and provided support and supervision to CBDs throughout the campaign, including checks for implementation fidelity which were done through unannounced spot checks (CBDs were informed these would happen during their training).

\section{Study design and modification}

The initial protocol was a factorial design ${ }^{23}$ whereby the 44 out of a possible 254 wards (clusters) were randomly allocated (1:1:1:1) to four arms. HIVST was distributed across all arms. All arms included fixed CBD stipends of US $\$ 50$ paid after an anticipated four to $4-6$ weeks of HIVST distribution and either: (i) conditional incentives for CBDs (US\$0.20 for each person who linked to post-test services at PSI outreach site), (ii) conditional incentives and demand-side (transport assistance) incentives for clients confirmed HIV positive to facilitate linkage to treatment, (iii) transport assistance for clients confirmed HIV positive and (iv) neither conditional incentives for CBDs nor transport assistance (figure 1).

The initial primary outcome was uptake of any health service at PSI clinics following distribution of self-test kits. However, on implementation (Mberengwa District), two major problems became apparent: first, transport assistance for recently diagnosed HIV patients had become standard of care, thus precluding evaluation of this intervention. Second, linkage data showed much higher than anticipated use of non-PSI clinics.

Hence, in consultation with our Technical Advisory Group (TAG), the demand-side incentive was dropped, and primary and secondary outcomes were redefined around endline survey data that did not require direct capture of clinic use. The six Mberengwa district clusters were dropped from analysis, leaving 38 wards in their original allocation for a two-arm clusterrandomised trial comparing fixed stipend only versus fixed stipend plus conditional incentives. The fixed stipend is standard practice for PSI community-based interventions.

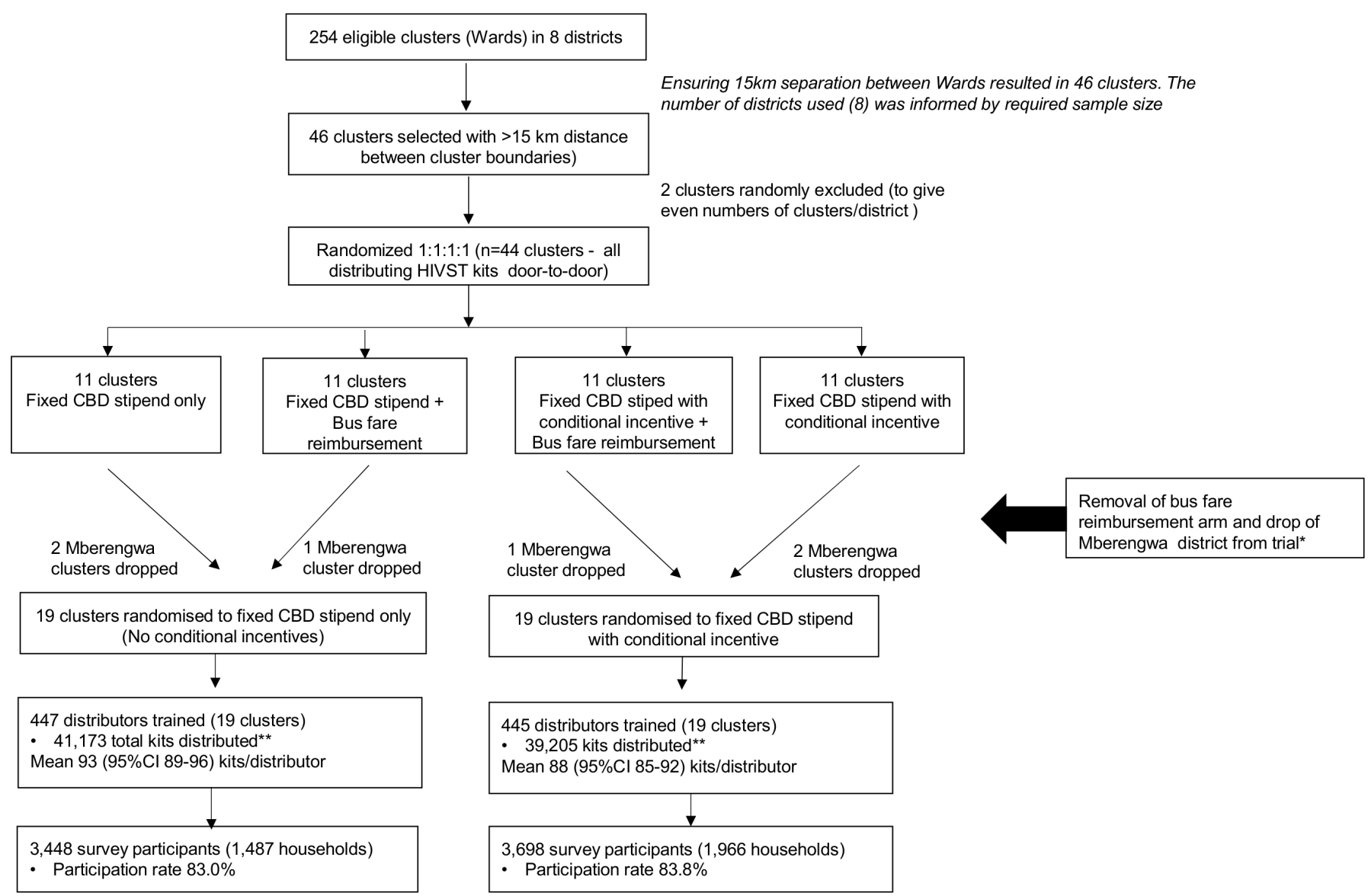

Figure 1 Trial profile. *The initial design was a 2x2 factorial trial with two interventions: conditional incentives for CBDs and bus fare reimbursements to support linkage to care for clients testing HIV positive. However, during implementation in the first six clusters (in one District, Mberengwa), transport support for clients testing positive became standard of care, thus precluding evaluation of the intervention. We therefore dropped the bus fare reimbursement intervention after consultation with the Technical Advisory Group. This left 38 clusters in seven districts with their distributor-incentive allocation intact. ${ }^{* *}$ No figures provided by one distributor in each arm. CBDs, community-based distributors 
The conditional incentive amount $(\$ 0.20$ per client attending PSI clinic services, including non-HIV services) was determined through consultation between researchers and programme implementers, aiming for an amount that was affordable and thus scalable, yet high enough to motivate desired behaviour but low enough to minimise the risk of unethical behaviour (such as forced testing, false clients) among CBDs. To give a sense of the value, at the time of the study, a loaf of bread cost $\$ 0.70$ $\$ 0.90$ and a local one-way bus ticket cost $\$ 0.20-\$ 0.50$.

Administration of incentives was based on counting appointment cards provided to each HIVST client that were redeemed by PSI mobile clinic staff. Client selfreports were also accepted from clients who could not produce appointment cards but had a convincing account of having self-tested, for example, requests for confirmatory testing and/or production of used kits. Since CBDs operated in defined geographic areas, the residence of the client could be used to identify the CBD.

In addition to the trial, we conducted a before-duringafter time-series analysis of ART initiations in clinic catchment areas receiving and not receiving HIVST, described below.

\section{Randomisation and masking}

Under the original study design, 44 clusters were randomly allocated to one of four arms. Randomisation was restricted by district and proximity to a health facility $^{24}$ using Stata V.15.1. From more than 7.5 billion ways of randomising 44 clusters to four arms, we randomly selected 100000 on which we applied the restriction criteria. This produced 32629 eligible allocations from which we randomly selected 1000 . During a public randomisation ceremony, we randomly selected one of these allocations.

Although distributors were not masked to study arm, clients receiving HIVST did not know that there were differences in the way distributors were paid, and all analyses used masked data (study arms were anonymised) with unmasking only after analysis of the primary outcome was finalised.

\section{Outcomes}

Population-based survey

The primary outcome was the proportion of survey participants who self-reported having accessed any clinic services from PSI outreach or local clinic visits since the start of HIVST kit distribution in their cluster, regardless of whether they had self-tested or not. This was assessed through response to the question 'Since about sixweeks ago (when CBDs came to your area to distribute self-test kits), have you been to a clinic or HIV testing facility for any service that you wanted for yourself?'. The outcome included non-HIV services such as hypertension, diabetes screening and family planning services to prevent unintended disclosure of HIV status.

Secondary outcomes were the proportion of survey participants self-reporting that they had:
1. Used an HIVST kit (all respondents).

2. Accessed confirmatory testing (for respondents who stated having self-tested positive only).

3. Initiated ART (for respondents who stated having selftested positive only).

4. Self-tested HIV-negative and had VMMC (for previously uncircumcised male respondents only).

\section{Outcome evaluation}

\section{Population-based surveys}

Six to 8 weeks after completion of HIVST kit distribution, a population-based survey was conducted in four randomly selected National Census Office Enumeration Areas (EA) in each cluster. Within each EA, $50 \%$ of households were randomly selected using Open Data Kit, ${ }^{25}$ and all members of selected households aged $\geq 16$ years were invited to participate, with written informed consent. Questionnaires used Audio Computer Assisted Self Interview (ACASI) on electronic tablets (male/female participants listened to male/female voices, respectively) and included demographic details; history of HIV testing and ART; access to, use and results of self-testing and uptake of any health services including confirmatory testing and ART following kit distribution.

The survey was conducted at population level (rather than restricted to individuals who received HIVST kits) because we wanted to determine HIVST uptake and population level impact of the interventions.

\section{ART clinic data extraction for before-during-after time series analysis}

Catchment areas (defined and published at health facilities) of all public sector facilities providing ART in each district were mapped in relation to wards of both trial arms and categorised into clinics with and without catchmentarea HIVST distribution. Numbers of ART initiations per month were extracted from clinic registers for the period 6 months before HIVST distribution, during distribution, and 3 months following completion of distribution. For clinics without catchment-area HIVST, we matched the before-during timepoints to those of the closest trial ward.

Time series evaluations can be affected by concurrent campaigns/initiatives, but during the trial, PSI were the only organisation partnering Ministry of Health to provide HIV testing services in the selected study districts (and were the only provider of HIVST in the country).

\section{Before-during-after study}

Differences in ART initiation rates for public sector health facilities were defined by time period (before, during and after) of HIVST distribution, using preintervention initiation rates as the baseline for each clinic, and comparing during-after time trends between facilities with and without catchment-area HIVST distribution. 


\section{Statistical methods}

Study power

Following protocol amendment to the two-arm design, the study power was recalculated, having initially been based on differences in demand for PSI outreach services. Using standard methodology, ${ }^{26}$ and assuming 200 postintervention survey participants per cluster, an intercluster coefficient of variation $(\mathrm{k})$ of 0.3 , and $10 \%$ uptake of any clinic service (as informed by a previous survey) among postintervention survey participants in the least effective arm, then 38 clusters would provide $>90 \%$ power to detect a $50 \%$ difference between arms, from an assumed $10 \%-15 \%$.

\section{Analysis of population-based survey data}

The statistical analysis plan was finalised for the two-arm (modified intention-to-treat) trial before data collection ended. Analysis used Stata V.15.1. ${ }^{27}$ We conducted logistic regression of individual-level data, with random effects adjustment to account for the cluster ${ }^{26}$ to estimate prevalence ratios and differences by arm, and their associated $95 \%$ CI. ${ }^{28}$ A permutation test incorporating the restriction criteria used for randomisation was used to estimate the primary outcome p-value.

The primary and secondary analyses were adjusted for imbalance by arm in a priori variables, with educational attainment, household food insecurity and mental distress meeting these criteria. Household food insecurity was defined by $\geq 2$ affirmative responses to three questions, one from each domain of the Household Food Insecurity Access Scale. ${ }^{29}$ Risk of mental distress was defined by a score of $\geq 9$ on the locally validated Shona Symptom Questionnaire. ${ }^{30}$

Sub-group analyses used data stratified by sex, age, food insecurity and mental distress. Sensitivity analyses, accounting for clustering at both the ward and household levels are shown in online supplemental appendix 2.

\section{Analysis of ART clinic data}

Generalised estimation equations were used to analyse relationships between ART initiation and campaign period, and to test for differences between clinics with and without catchment-area HIVST distribution, and between trial arms. We fitted separate models assessing rate differences and rate ratios. For both models, we assumed ART initiations were Poisson distributed. In both analyses, we adjusted for district (fixed effect), observation days (offset), preintervention clinic testing rates (average number of post-test counselling sessions per month, modelled as quadratic) and calendar month (fixed effect).

\section{Ethical considerations and governance}

As the conditional incentive intervention carried minimal risk to participants, no interim analyses were planned. An independent TAG met every 6 months to review progress.

\section{Patient and public involvement}

Before the trial, we conducted formative research including qualitative studies and discrete choice experiments where we obtained communities' preferences for door-to-door distribution of HIV self-tests. ${ }^{41}$ We used these findings to refine the HIVST distribution strategy. During the trial, as part of community entry activities, the study team met with community leaders in each ward to seek their consent for involvement of their communities and their guidance in the selection of CBDs. At the end of the study, community meetings were held to discuss research findings and get views on what worked well/less well during the intervention.

\section{Role of the funding source}

The funder did not influence the study design, protocol writing process, implementation decisions, data analysis and data interpretation. The corresponding author has full access to data.

\section{RESULTS}

Between August 2016 and August 2017, 445 CBDs in the conditional incentive arm and 447 CBDs in the no incentive arm distributed a mean of 88 (CI: 85 to 92) and 93 (CI: 89 to 96 ) kits each, respectively. The total distributed per arm was 39205 and 41173 (figure 1). Populations sizes were similar by arms, with harmonic means of 3993 (CI: 2881 to 6501) and 4796 (CI: 4020 to 5944) in HIVST and non-HIVST communities, respectively (based on 2012 census data). Distribution was carried out over 19 days (range 19-25 days) per cluster, with a median of 2313 (IQR: 1403-2521) kits distributed per cluster in the conditional incentive arm and 2071 (1646-2616) in the no incentives arm. In total, $171(38.4 \%)$ CBDs in conditional incentive arm were given conditional incentives, with incentive amounts ranging from $\$ 0.20$ to $\$ 6.00$ (median \$0.80), corresponding to 1-30 clients linked per CBD.

The postintervention survey included 7146 participants from 3813 households, with a response rate of $83.8 \%$ in the conditional incentive arm and $83.0 \%$ in the no incentive arm. Participant characteristics were largely comparable between arms (table 1), although small imbalances by arm in food insecurity, education and mental distress were adjusted for in subsequent analyses.

Overall, $6335(88.7 \%)$ of participants had ever tested for HIV (table 2), and 5838 (81.7\%) had ever heard of HIVST. Of note, $4039(56.5 \%)$ of those who had heard of HIVST had taken a self-test kit during HIVST distribution, and 3593 (89.0\% of kit recipients) reported having self-tested. The overall population uptake of HIVST was $50.3 \%$ (3593/7146). Uptake varied relatively little by arm, age or sex, with $46.2 \%$ of young people (age 16-25 years) and $46.5 \%$ of men reporting having self-tested. Among self-testers, $36.3 \%(1305 / 3593)$ reported that HIVST was their first ever HIV test; this represented $18.3 \%$ of the overall sample. 
Table 1 Characteristics of respondents in household surveys

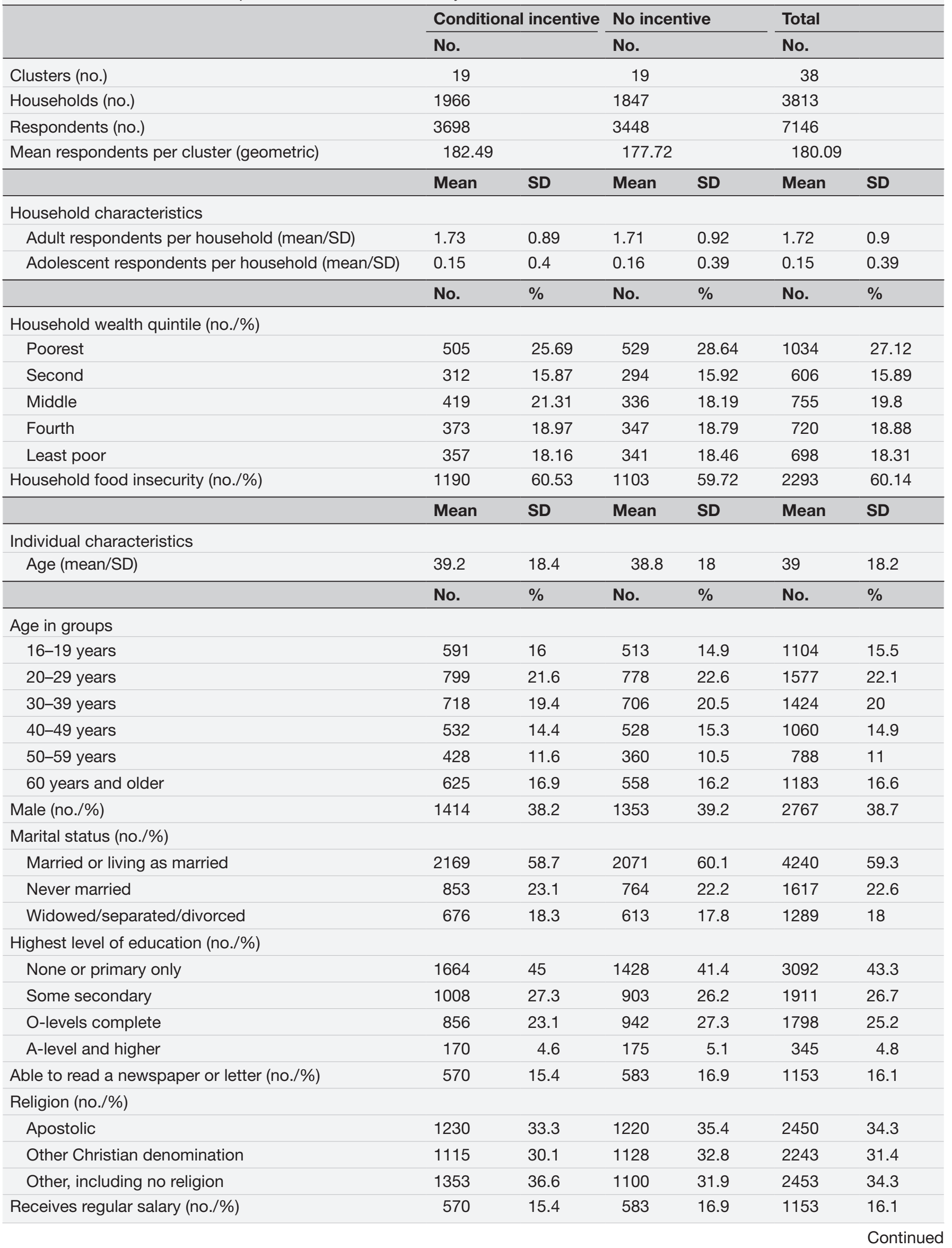


Table 1 Continued

\begin{tabular}{lrrrrrr}
\hline & No. & \% & No. & $\%$ & No. & $\%$ \\
\hline SSQ $^{*}=9$ (no./\%) & 1599 & 43.2 & 1622 & 47 & 3221 & 45.1 \\
\hline Ever tested for HIV (no./\%) & 3236 & 87.5 & 3099 & 89.9 & 6335 & 88.7 \\
\hline Self-reported HIV+ (no./\%) & 450 & 12.2 & 377 & 10.9 & 827 & 11.6 \\
\hline Self-reported current ART use, among HIV+ (no./\%) & 388 & 86.2 & 319 & 84.6 & 707 & 85.5
\end{tabular}

*Shona Symptom Questionnaire.

ART, antiretroviral therapy.

\section{Effect of conditional incentives \\ Uptake of HIVST}

There were 1770 (47.9\%) and 1823 (52.9\%) participants who reported self-testing in the conditional incentives and no incentives arms, respectively, giving an adjusted prevalence ratio (aPR) 0.91 (95\% CI: 0.80 to 1.02 ).

Uptake of post-HIVST distribution services (primary outcome)

We found no evidence of a difference in uptake of postHIVST services by arm with $1062(28.7 \%)$ and 1075 $(31.2 \%)$ clients in conditional incentive and no incentive arm, respectively, linked to any service $(\mathrm{aPR}=0.94$; $95 \%$ CI: 0.86 to 1.03 , permutation test $\mathrm{p}=0.13$; table 3 ). The majority of participants who linked (1768/2137; $82.7 \%$ ) visited the local clinic/hospital. The most commonly sought post-test services from the local clinic/ hospital (multiple responses possible) were HIV testing and counselling $(38.6 \%)$, treatment for an ailment $(16.7 \%)$, routine clinic visit or prescription refill $(12.2 \%)$, HIV care-CD4 cell count (12.1\%) and family planning services $(11.0 \%)$. Subgroup analysis showed no evidence of a difference in linkage to post-test services by arm, by gender, age, food insecurity or mental distress (online supplemental appendix 3). The cluster coefficient of variation, $\mathrm{k}$, for uptake of post-test services was 0.05 .

\section{Other outcomes}

We did not find evidence of a difference by arm for our secondary outcomes of linkage to PSI outreach services (table 3). The preset analyses of confirmatory testing and uptake of ART for participants with reactive self-tests were dominated by retesting while already on ART (table 3), which was not the intention of this intervention. As such, we also present a post-hoc analysis excluding respondents who reported they were already on ART at the time of HIVST (table 3). This provides some evidence that individuals with newly diagnosed HIV were more likely to have attended for confirmatory testing in the conditional incentives arm $(23 / 33,75.8 \%)$ compared with the no incentive arm $(20 / 40,50.0 \%)$ : aPR $1.59 ; 95 \%$ CI 1.05 to 2.39 .

\section{Effect of HIVST on ART clinic initiations}

To determine the effect of HIVST on ART initiations, we extracted data from all 164 health facilities that provided ART in the study districts. Of these, 40 facilities served the 38 HIVST communities and recorded 3138 ART initiations, while 124 facilities were outside the catchmentarea for HIVST distribution and reported 9670 ART initiations. Our data show that the average number of ART initiations per clinic day preintervention was similar between HIVST and non-HIVST health facilities. During the period of HIVST distribution, there was a difference in rate of ART initiations between HIVST distribution and non-HIVST areas; this difference disappeared after completion of HIVST distribution. Online supplemental appendix 4A summarises the number of ART initiations per month during the three periods before, during and after HIVST distribution in HIVST and non-HIVST communities: in HIVST communities, the number of ART initiations increased during the distribution period

Table 2 Coverage of self-testing after distribution

\begin{tabular}{llll}
\hline Characteristic & Conditional incentives & No incentives & Total \\
\hline Respondents - number & 3698 & 3448 & 7146 \\
\hline Ever tested for HIV & $3236 / 3698(87.5 \%)$ & $3099 / 3448(89.9 \%)$ & $6335 / 7146(88.7 \%)$ \\
\hline Ever heard of HIV self-testing & $2962 / 3698(80.1 \%)$ & $2876 / 3448(83.4 \%)$ & $5838 / 7146(81.7 \%)$ \\
\hline Received kit during intervention & $1983 / 3698(53.6 \%)$ & $2056 / 3448(59.6 \%)$ & $4039 / 7146(56.5 \%)$ \\
\hline Used kit to self-test & $1770 / 3698(47.9 \%)$ & $1823 / 3448(52.9 \%)$ & $3593 / 7146(50.3 \%)$ \\
\hline \% First-time testers & $642 / 3698(17.4 \%)$ & $663 / 3698(19.2 \%)$ & $1305 / 7146(18.3 \%)$ \\
\hline Overall coverage of self-testing & $1770 / 3698(47.9 \%)$ & $1823 / 3448(52.9 \%)$ & $3593 / 7146(50.3 \%)$ \\
\hline Coverage among men & $628 / 1414(44.4 \%)$ & $658 / 1353(48.6 \%)$ & $1286 / 2767(46.5 \%)$ \\
\hline Coverage among aged $<25$ years & $463 / 1037(44.7 \%)$ & $456 / 952(47.9 \%)$ & $919 / 1989(46.2 \%)$
\end{tabular}




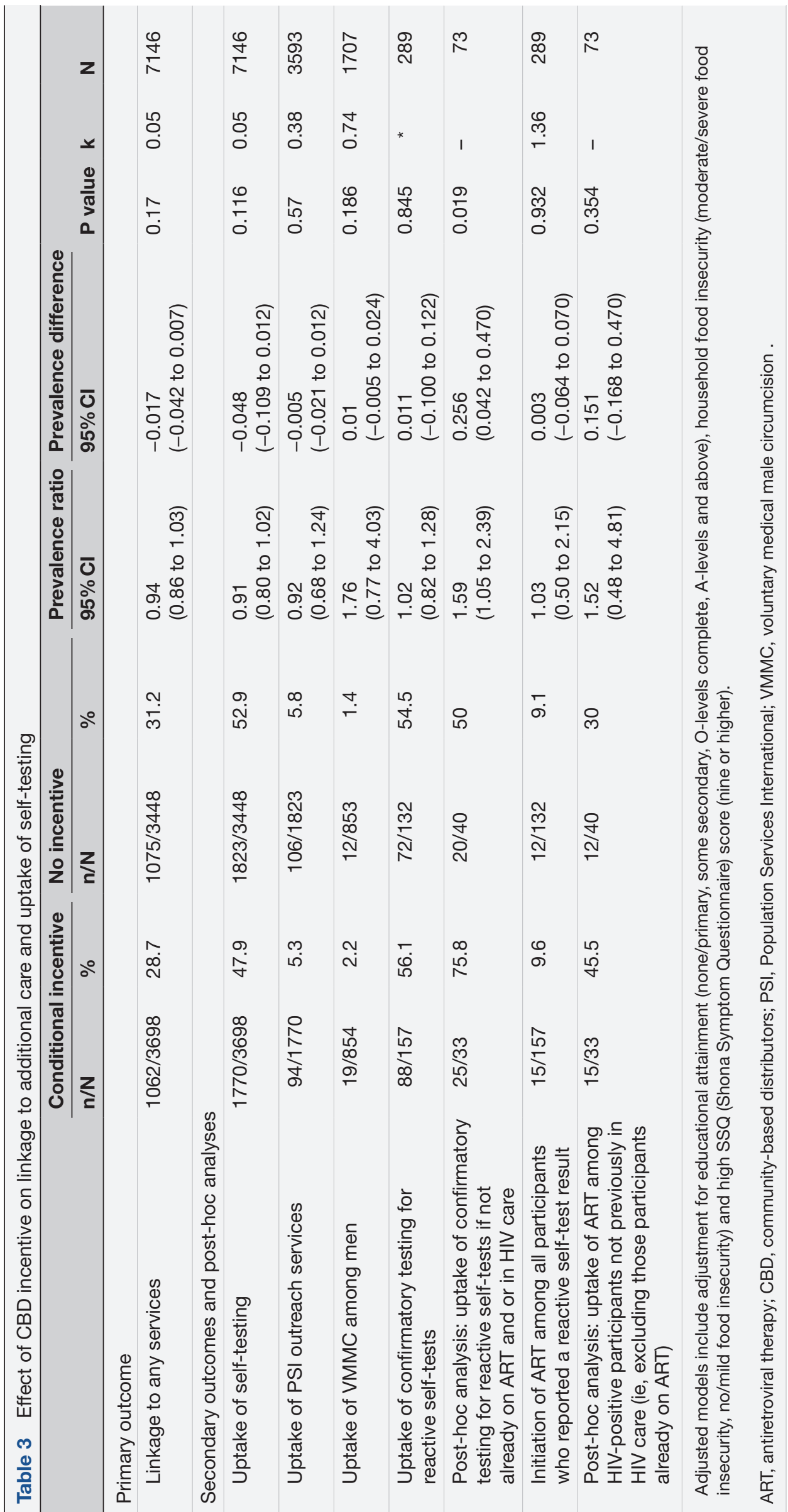

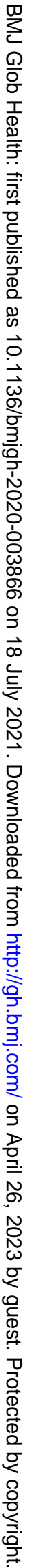




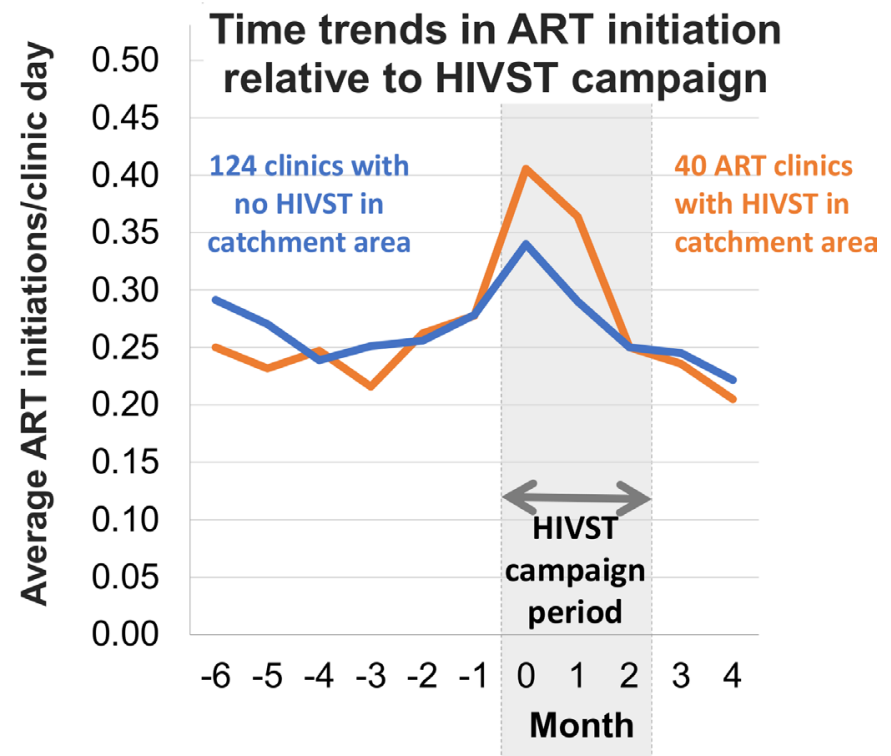

Figure 2 Trends in ART initiation rates before, during and after the HIVST campaign period.

from 7.31 to 9.59 initiations per month, then decreased thereafter to 6.53 initiations per month.

Figure 2 illustrates the change in ART initiation rates over time and interaction with HIVST, with the increase in ART initiation rates in HIVST communities corresponding to an adjusted ART initiation rate ratio of 1.27 (95\% CI: 1.13 to 1.42). Details are given in online supplemental appendix 4B. Once HIVST distribution ended, the adjusted initiation rate ratio was 1.02 (95\% CI: 0.88 to 1.18) compared with the preintervention period. There was no difference in ART initiation by trial arm postHIVST distribution (data not shown).

\section{DISCUSSION}

In this pragmatic cluster-randomised trial, we found that community-based HIVST campaigns achieved high uptake of testing across both arms, including groups with historically low testing rates (men, young people) and those who had not tested previously. Using a separate quasi-experimental design, we showed that HIVST campaigns in both arms were associated with a $27 \%$ relative increase in ART initiation rates at nearby public health facilities, with return to pre-HIVST rates as soon as the campaign was over. There was, however, no evidence of additional benefit from providing kit distributors with a small linkage incentive (US $\$ 0.20$ per client linked) on top of a fixed stipend, with no evidence of a difference between arms on uptake of post-test services including ART or on HIVST uptake. Uptake of confirmatory testing was higher for newly diagnosed HIV-positive participants in the conditional incentive arm, but this finding was based on small numbers and was a post-hoc modification to the planned analysis to exclude clients who were using HIVST to re-test while on ART.
Our results add to the growing body of evidence showing that HIVST can increase uptake of HIV testing. ${ }^{15}$ Following HIVST distribution, $88.7 \%$ of participants surveyed reported ever testing for HIV, compared with $75 \%$ in the nationally representative Zimbabwe Public Health Impact Assessment conducted in the same year. ${ }^{12}$ These data suggest that campaign style HIVST distribution can bring about a step change in knowledge of HIV status, with good uptake by men, young people and a substantial proportion being first-time testers. Of note, we found an increase in ART initiation immediately after commencement of HIVST distribution, with rates going back to previous levels after distribution stopped, showing the value of self-testing in achieving timely linkage.

Studies of community-based provider testing have shown longer times to linkage than suggested by our ART clinic-level data, ${ }^{32}$ for instance in the HIV Prevention Trials Network 071 trial (known as POPART) in Zambia and South Africa, the median time to linkage was 6 months. Data from our survey, conducted just 6 weeks after the HIVST campaign data, add support to more rapid linkage with most previously undiagnosed selftesters with reactive results having already confirmed them. It is plausible that reactive (positive) self-tests may tend to prompt more rapid action than reactive providertests, given the additional uncertainties regarding the result and likely desire for experienced in-person posttest counselling. Not only are reactive self-tests preliminary (unconfirmed by a second or third test kit), but self-testers acknowledge their own inexperience as a motivator for clinic confirmation when they get unexpected results. $^{34}$

A small conditional incentive of US $\$ 0.20$ per person taking up post-test services did not increase linkage overall although confirmatory testing of newly diagnosed HIV-positive HIVST clients appeared to have been increased. Existing literature has shown the effectiveness of demand-side incentives on engagement along the HIV cascade, including increasing linkage to HIV care and prevention following self-testing among male partners of women attending antenatal care clinics in Malawi. ${ }^{19}$ Importantly, the Malawi trial showed a dose-effect with US $\$ 10$ more effective than US\$3 in prompting postHIVST clinic attendance. ${ }^{19}$ In contrast, uptake of HIVST by male partners, which was already $93 \%$ in the no-incentive arm-implying strong inherent motivation, was unaffected by incentivisation. ${ }^{19}$ Motivation was high in both arms of the current trial, with CBDs reporting pride in delivering an important and novel intervention to their community. ${ }^{35}$ Motivation was also potentially driven by the $\$ 50$ base amount. We could not test a range of amounts for the conditional incentive due to time pressure and acknowledge that the amount may simply have been too low. We were constrained to test a small amount that was potentially scalable but with low risk of unintended consequences.

Measuring linkage is already challenging for standard provider-delivered HIV testing, but becomes more so 
with self-testing with loss of provider-control over the test results. ${ }^{36}$ Attempts to track cohorts of HIV self-testers and link individual HIVST clients to confirmatory testing and ART initiation events, as occurs for monitoring standard HIV testing services, may be futile. Even using more approximate triangulation approaches looking at initiation rates at health facilities may be undermined by clients failing to disclose previous testing episodes and results. Additionally, some clients may opt to initiate treatment in a different location from where they initially tested positive to maintain confidentiality. The lack of national unique patient identifiers which can be used to track individuals across facilities coupled with poor penetration of electronic medical record systems in many African settings adds to this challenge. Despite its limitation, the approach used here to infer linkage to care following HIVST through a time-series analysis, provides an effective, low cost alternative to evaluating the impact of HIVST, circumventing the difficulties of measuring linkage more directly, and is one that other programmes could consider if using similar high intensity campaigns aimed at high coverage.

The major strength of this study was the large-scale implementation of community-based HIVST, providing evidence on feasibility, coverage and linkage. The accompanying time-trend analysis showing increased clinic-level ART initiations associated with HIVST adds powerful support for HIVST-related health outcomes using methods that bypass the need for cohort tracking despite also showing no difference between incentive trial arms. PSI was the sole HIV testing partner operating in the study districts, allowing us to confidently exclude non-study interventions during the HIVST campaign period.

Limitations include potential bias from trial outcomes based on self-report, although we used ACASI to minimise this risk. ${ }^{37}$ The trial deviated from the original design (see Methods section), dropping one of the two planned interventions (demand-side incentives) and redefining the way that the primary outcome was to be captured, which necessitated removal of one district (six clusters) from analysis postrandomisation. As randomisation was restricted by district, we retained balance between arms in the remaining clusters. People already on ART using HIVST to retest, which is common for a variety of reasons, ${ }^{8} 3839$ outnumbered new HIV diagnoses in our endline survey necessitating post-hoc adaptation of confirmatory testing outcome. Retesting on ART has become more problematic for evaluation of routine HIV testing services, regionally, as countries approach 90-90-90 targets, and should be discouraged as far as possible. We acknowledge that our conclusions regarding conditional incentives would have been more robust had we been able to include prior dose-ranging studies. For the difference-in-differences analysis, clinic catchment areas were larger than HIVST clusters and not all newly diagnosed PLHIV will choose their local clinic for HIV services: one explanation for the smaller peak seen for designated non-HIVST clinics during HIVST distribution (figure 2). As such, we may have underestimated the effect of HIVST campaigns on demand for ART.

In summary, community-based HIVST campaigns achieved a step change in uptake of HIV testing, including among men and young people who typically have poor testing uptake. HIVST was accompanied by a significant increase in clinic-based ART initiations during the campaign period. Delivery of HIVST by briefly trained community members was feasible, but a conditional financial incentive added little to their performance over a fixed stipend to distribute this novel technology, suggesting either high levels of inherent nonmonetary motivators, or a subeffective incentive amount or a combination of these two. Given our post-hoc analysis showing higher linkage to confirmatory testing for newly diagnosed HIV-positive clients in the conditional incentive arm, future research should aim to optimise the nature and size of incentives to increase uptake and linkage while weighing the benefits and costs as highlighted in WHO guidance. ${ }^{40}$

\section{Author affiliations}

${ }^{1}$ CeSHHAR Zimbabwe, Harare, Zimbabwe

${ }^{2}$ Department of International Public Health, Liverpool School of Tropical Medicine, Liverpool, UK

${ }^{3}$ MRC Tropical Epidemiology Group, London School of Hygiene \& Tropical Medicine, London, UK

${ }^{4} \mathrm{HIV}$ and Tuberculosis, Population Services International Global, Washington, DC, USA

${ }^{5}$ Department of Sexual Reproductive Health Rights and Innovations, Population Services International Zimbabwe, Harare, Zimbabwe

${ }^{6}$ Women's Global Health Imperative, RTI International, Berkeley, California, USA

${ }^{7} \mathrm{HIV}$ and TB Unit, Ministry of Health and Child Care, Harare, Zimbabwe

${ }^{8}$ Department of Global Health and Development, Faculty of Public Health and Policy, London School of Hygiene and Tropical Medicine, London, UK

${ }^{9}$ HIV Department, Geneva, Switzerland

${ }^{10}$ Department of Infectious Disease Epidemiology, Faculty of Epidemiology and Population Health, London School of Hygiene \& Tropical Medicine, London, UK

${ }^{11}$ Department of Clinical Research, Faculty of Infectious and Tropical Diseases, London School of Hygiene and Tropical Medicine Faculty of Epidemiology and Public Health, London, UK

${ }^{12}$ Malawi-Liverpool Wellcome Trust Clinical Research Programme, Blantyre, Malawi

Twitter Euphemia Lindelwe Sibanda @Euphemia4, Collin Mangenah @cmangenah01, Miriam Taegtmeyer @MiriamTaegtmeye and Cheryl Johnson @ccasejohn

Contributors Formulated the research study and design: FMC, EC, MTa, KH, CJ, ELS, GN, KLF, HAW, MN, FT-P. Informed development of data collection methods and collected data: FMC, EC, MTu, MTa, MN, JD, MNM, ELS, GN, CW. Analysed the data or contributed to the analysis: MN, ELS, HAW, KLF. Substantially provided intellectual input to the manuscript: FMC, EC, HAW, KH, FT-P, KLF, MTu, MTa, CJ, MN, MNM, CM, SN, CW.

Funding This study was funded by Unitaid (STAR Initiative), Sub agreement number 4214-CeSHHAR. ELC is funded by Wellcome Trust (WT200901/Z/16/Z). We thank all participants and staff, and the STAR technical advisory group (Peter Godfrey-Faussett, Ruanne Barnabas, Vincent Wong, Mohammed Lamorde).

Map disclaimer The depiction of boundaries on this map does not imply the expression of any opinion whatsoever on the part of BMJ (or any member of its group) concerning the legal status of any country, territory, jurisdiction or area or of its authorities. This map is provided without any warranty of any kind, either express or implied.

\section{Competing interests None declared.}

Patient consent for publication Not required. 
Ethics approval Ethical approval was obtained from Medical Research Council of Zimbabwe (MRCZ/A/2038), University College London Ethics Committee (6084/004) and London School of Hygiene and Tropical Medicine Ethics Committee (11738). The trial was registered with Pan African Clinical Trial Registry (PACTR201607001701788).

Provenance and peer review Not commissioned; externally peer reviewed.

Data availability statement Data are available upon request. De-identified data are available from the corresponding author upon request.

Supplemental material This content has been supplied by the author(s). It has not been vetted by BMJ Publishing Group Limited (BMJ) and may not have been peer-reviewed. Any opinions or recommendations discussed are solely those of the author(s) and are not endorsed by BMJ. BMJ disclaims all liability and responsibility arising from any reliance placed on the content. Where the content includes any translated material, BMJ does not warrant the accuracy and reliability of the translations (including but not limited to local regulations, clinical guidelines, terminology, drug names and drug dosages), and is not responsible for any error and/or omissions arising from translation and adaptation or otherwise.

Open access This is an open access article distributed under the terms of the Creative Commons Attribution IGO License (CC BY NC 3.0 IGO), which permits use, distribution,and reproduction in any medium, provided the original work is properly cited. In any reproduction of this article there should not be any suggestion that WHO or this article endorse any specific organization or products. The use of the WHO logo is not permitted. This notice should be preserved along with the article's original URL.

Disclaimer: The author is a staff member of the World Health Organization. The author alone is responsible for the views expressed in this publication and they do not necessarily represent the views, decisions or policies of the World Health Organization.

\section{ORCID iDs}

Euphemia Lindelwe Sibanda http://orcid.org/0000-0003-1754-1076

Melissa Neuman http://orcid.org/0000-0002-8870-6504

Collin Mangenah http://orcid.org/0000-0002-0733-0622

Frances M Cowan http://orcid.org/0000-0003-3087-4422

\section{REFERENCES}

1 UNAIDS. Global AIDS update 2019: communities at the centre. Geneva, Switzerland: UNAIDS, 2019.

2 World health Organisation. Guidelines on HIV self-testing and partner notification: supplement to consolidated guidelines on HIV testing services. Geneva, Switzerland, 2016.

3 Figueroa C, Johnson C, Verster A, et al. Attitudes and acceptability on HIV Self-testing among key populations: a literature review. AIDS Behav 2015;19:1949-65.

4 Indravudh PP, Sibanda EL, d'Elbée M, et al. 'I will choose when to test, where I want to test': investigating young people's preferences for HIV self-testing in Malawi and Zimbabwe. AIDS 2017;31(Suppl 3):S203-12.

5 Krause J, Subklew-Sehume F, Kenyon C, et al. Acceptability of HIV self-testing: a systematic literature review. BMC Public Health 2013;13:735.

6 Mokgatle MM, Madiba S. High acceptability of HIV Self-Testing among technical vocational education and training college students in Gauteng and North West Province: what are the implications for the scale up in South Africa? PLoS One 2017;12:e0169765.

7 Napierala Mavedzenge S, Sibanda EL, Mavengere Y. Acceptability, feasibility, and preference for HIV self-testing in Zimbabwe. Durban, South Africa: IAS, 2016

8 Choko AT, MacPherson P, Webb EL, et al. Uptake, accuracy, safety, and linkage into care over two years of promoting annual SelfTesting for HIV in Blantyre, Malawi: a community-based prospective study. PLoS Med 2015;12:e1001873.

9 Napierala-Mavedzenge S, Sibanda EL, Mavengere Y. Supervised HIV self-testing to inform implementation and scale up of self-testing in Zimbabwe. The 8th IAS Conference on HIV Pathogenesis, Treatment and Prevention (IAS 2015); Jul 2015, Vancouver, Canada: IAS, 2015.

10 Pant Pai N, Sharma J, Shivkumar S, et al. Supervised and unsupervised self-testing for HIV in high- and low-risk populations: a systematic review. PLoS Med 2013;10:e1001414.

11 UNAIDS. Global AIDS update: Miles to go - closing gaps, breaking barriers, righting injustices. Geneva, Switzerland: UNAIDS, 2018.

12 Zimbabwe Ministry of Health and Child Care. Zimbabwe populationbased HIV impact assessment (ZIMPHIA). Harare, Zimbabwe: MOHCC, 2016.
13 UNAIDS. Zimbabwe country data Geneva. Switzerland: UNAIDS, 2018. http://www.unaids.org/en/regionscountries/countries/ zimbabwe

14 Cowan FM, Davey C, Fearon E, et al. Targeted combination prevention to support female sex workers in Zimbabwe accessing and adhering to antiretrovirals for treatment and prevention of HIV (SAPPH-IRe): a cluster-randomised trial. Lancet HIV 2018;5:e417-26.

15 Johnson CC, Kennedy C, Fonner V, et al. Examining the effects of HIV self-testing compared to standard HIV testing services: a systematic review and meta-analysis. J Int AIDS Soc 2017;20:21594.

16 Unitaid, World Health Organisation. Market technology landscape: HIV rapid diagnostic tests for self-testing. Geneva, Switzerland: Unitaid, WHO, 2018.

17 Cambiano V, Ford D, Mabugu T, et al. Assessment of the potential impact and cost-effectiveness of Self-Testing for HIV in low-income countries. J Infect Dis 2015;212:570-7.

18 MacPherson P, Lalloo DG, Webb EL, et al. Effect of optional home initiation of HIV care following HIV self-testing on antiretroviral therapy initiation among adults in Malawi: a randomized clinical trial. JAMA 2014;312:372-9.

19 Choko AT, Corbett EL, Stallard N, et al. HIV self-testing alone or with additional interventions, including financial incentives, and linkage to care or prevention among male partners of antenatal care clinic Attendees in Malawi: an adaptive multi-arm, multi-stage cluster randomised trial. PLoS Med 2019;16:e1002719.

20 Sharma M, Ying R, Tarr G, et al. Systematic review and metaanalysis of community and facility-based HIV testing to address linkage to care gaps in sub-Saharan Africa. Nature 2015;528:S77-85.

21 Bassett IV, Wilson D, Taaffe J, et al. Financial incentives to improve progression through the HIV treatment cascade. Curr Opin HIV AIDS 2015;10:451-63.

22 STAR Project. HIV Self-testing Africa project. London, United Kingdom, 2017. http://hivstar.Ishtm.ac.uk/

23 Montgomery AA, Peters TJ, Little P. Design, analysis and presentation of factorial randomised controlled trials. BMC Med Res Methodol 2003;3:26.

24 Sismanidis $\mathrm{C}$, Moulton $\mathrm{LH}$, Ayles $\mathrm{H}$, et al. Restricted randomization of ZAMSTAR: a $2 \times 2$ factorial cluster randomized trial. Clin Trials 2008;5:316-27

25 Open Data Kit. Open Data Kit website [website], 2019. Available: https://opendatakit.org/

26 Hayes RJ, Moulton LH. Cluster randomised trials. Boca Raton, Florida: CRC Press, 2009.

27 StataCorp LLC. StatalC College Station. Texas: StataCorp LLC 1985-2019.

28 Williams R. Using the margins command to estimate and interpret adjusted predictions and marginal effects. Stata J 2012;12:308-31.

29 Deitchler M, Ballard T, Swindale A. Validation of a measure of household hunger for CrossCultural use. 360. Washington, DC: FHI, 2010.

30 Patel V, Simunyu E, Gwanzura F, et al. The Shona symptom questionnaire: the development of an Indigenous measure of common mental disorders in Harare. Acta Psychiatr Scand 1997;95:469-75

31 Sibanda EL, d'Elbée M, Maringwa G, et al. Applying user preferences to optimize the contribution of HIV self-testing to reaching the "first 90" target of UNAIDS Fast-track strategy: results from discrete choice experiments in Zimbabwe. J Int AIDS Soc 2019;22(Suppl 1):e25245.

32 Seeley J, Bond V, Yang B. Understanding the time needed to link to care and start art in seven HPTN 071 (PopART) study communities in Zambia and South Africa. AIDS Behav 2018.

33 SEARCH (Sustainable East Africa Research in Community Health). Search (sustainable East Africa research in community health). AIDS. 2018. Amsterdam, Netherlands: International AIDS Society, 2018.

34 Kumwenda MK, Corbett EL, Choko AT, et al. Post-test adverse psychological effects and coping mechanisms amongst HIV selftested individuals living in couples in urban Blantyre, Malawi. PLOS One 2019;14:e0217534.

35 Madanhire C, Ruhode N, Sibanda EL. Door-to-door distribution of HIV self-test kits: a qualitative study among community-based test kit distributors in Zimbabwe. Abidjan, Cote d' Ivoire: ICASA, 2017: 2017

36 Neuman M, Taegtmeyer M, Hatzold K, et al. Challenges in measurement of linkage following HIV self-testing: examples from the StAR project. J Int AIDS Soc 2019;22(Suppl 1):e25238.

37 Langhaug LF, Cheung YB, Pascoe SJS, et al. How you ask really matters: randomised comparison of four sexual behaviour questionnaire delivery modes in Zimbabwean youth. Sex Transm Infect 2011:87:165-73. 
38 Johnson CC, Fonner V, Sands A, et al. To err is human, to correct is public health: a systematic review examining poor quality testing and misdiagnosis of HIV status. J Int AIDS Soc 2017;20:21755.

39 Fuente-Soro L, Lopez-Varela E, Augusto O, et al. Monitoring progress towards the first UNAIDS target: understanding the impact of people living with HIV who re-test during HIV-testing campaigns in rural Mozambique. J Int AIDS Soc 2018;21:e25095.

40 World Health Organisation. Consolidated guidelines on HIV testing services for a changing epidemic. Geneva. 2019. Switzerland: WHO, 2019. 